\title{
Neural Network Observer-Based Finite-Time Formation Control of Mobile Robots
}

\author{
Caihong Zhang, ${ }^{1}$ Tairen Sun, ${ }^{2,3}$ and Yongping $\operatorname{Pan}^{4}$ \\ ${ }^{1}$ School of Electrical Engineering, Guangdong Mechanical and Electrical College, Guangzhou 510550, China \\ ${ }^{2}$ Key Laboratory of Autonomous Systems and Networked Control, Ministry of Education, \\ South China University of Technology, Guangzhou 510640, China \\ ${ }^{3}$ School of Electrical and Information Engineering, Jiangsu University, Zhenjiang 212013, China \\ ${ }^{4}$ School of Biomedical Engineering, National University of Singapore, Singapore 117575
}

Correspondence should be addressed to Yongping Pan; sbiepany@nus.edu.sg

Received 22 March 2014; Accepted 18 June 2014; Published 7 July 2014

Academic Editor: Simin Yu

Copyright (C) 2014 Caihong Zhang et al. This is an open access article distributed under the Creative Commons Attribution License, which permits unrestricted use, distribution, and reproduction in any medium, provided the original work is properly cited.

\begin{abstract}
This paper addresses the leader-following formation problem of nonholonomic mobile robots. In the formation, only the pose (i.e., the position and direction angle) of the leader robot can be obtained by the follower. First, the leader-following formation is transformed into special trajectory tracking. And then, a neural network (NN) finite-time observer of the follower robot is designed to estimate the dynamics of the leader robot. Finally, finite-time formation control laws are developed for the follower robot to track the leader robot in the desired separation and bearing in finite time. The effectiveness of the proposed NN finite-time observer and the formation control laws are illustrated by both qualitative analysis and simulation results.
\end{abstract}

\section{Introduction}

In recent years, the formation problem of mobile robots has attracted great attention. The contributions to formation control have been proposed mainly based on some approaches such as behavior-based ones [1, 2], virtual structure [3, 4], graph theory $[5,6]$, and leader-following [7-13]. Among these approaches, leader-following is widely used owing to its simplicity, scalability, and reliability. Yet, most of current methods on leader-following formation control for mobile robots are based on kinematic models [7-13], which results in the requirement of perfect velocity tracking.

Recently, leader-following formation control methods based on dynamic model have been proposed in [14-17], where the dynamics of the leader, such as the leader's pose (i.e., the position and direction angle) and velocity, becomes an important part of the formation control for its follower robots. In [14], leader-following formation was constructed based on the assumption that the leader communicates its pose, velocity vector, and the derivative of the velocity vector to its follower robot. In [15], to achieve leader-following formation tracking, RISE feedback control laws were designed for the follower robots under the strict assumptions that (1) the leader and follower's dynamics are sufficiently smooth and (2) the leader's pose, velocity, and the control law for the leader are communicated to the follower robots. Note that the communicating information needed in $[14,15]$ is of big quantity. In [16], to reduce the communicating information in the leader-follower formation, formation control was designed under the assumption that the separation and bearing can be measured by the follower robots and the leader robot communicates its orientation angle to its followers. However, the measurements of the separation and bearing by the follower robots are very difficult. In [17], a projection algorithm was used to estimate the velocity of the leader robot and leaderfollowing formation control laws were designed for the following robots based on a dynamic surface control technique. Yet, the communicating information of the leader's dynamics in [17] is required to be as little as possible, for the existence of cost and possible information loss in the information communication between the leader and its followers.

All the above mentioned results for the formation control are about asymptotic convergence. In other words, the formations in [1-17] cannot be achieved in finite time. However, 
finite-time formation control may be more attractive for the advantages of finite time control: higher convergence rates, higher accuracy, better disturbance rejections, and so on [1821]. In this paper, based on our previous work in [22], we consider the finite-time leader-following formation control for nonholonomic mobile robots based on their dynamic models, where the dynamics of the leader robot is unknown to the follower robot and only the pose of the leader can be obtained by the follower robot. The procedure of control design is as follows: first, the leader-following formation is transformed into special trajectory tracking; second, a neural network $(\mathrm{NN})$ finite-time observer of the follower robot is designed to estimate the dynamics of the leader robot; finally, finite-time formation control laws are developed for the follower robot to track the leader robot in the desired separation and bearing in finite time.

The rest of this paper is organized as follows. Section 2 introduces the dynamics of the leader and follower nonholonomic mobile robots, the radial basis function (RBF) $\mathrm{NN}$, and some results on finite-time control. A NN finitetime observer is used to estimate the leader's dynamics in Section 3.1 and the finite-time leader-following formation control is designed in Section 3.2. Simulation results are presented in Section 4. Finally, conclusions are given in Section 5 .

\section{Preliminary}

2.1. Dynamic Model. The dynamics of the nonholonomic mobile robots can be described as follows [22]:

$$
\begin{gathered}
M_{j}\left(q_{j}\right) \ddot{q}_{j}+V_{m j}\left(q_{j}, \dot{q}_{j}\right) \dot{q}_{j}+G_{j}\left(q_{j}\right) \\
=B_{j}\left(q_{j}\right) \tau_{j}+A_{j}^{T}\left(q_{j}\right) \lambda_{j},
\end{gathered}
$$

where $j=l, f, j=l$ is the subscript of the leader of the formation, $j=f$ is the subscript of the $i$ th follower of the leader, $q_{j}=\left[x_{j}, y_{j}, \theta_{j}\right]^{T}$ are the poses of the point located a distance $d_{j}$ from the rear axle, $\left[x_{j}, y_{j}\right]$ are the coordinates of the point, $\theta_{j}$ are the heading directions of the robot, $M_{j}\left(q_{j}\right) \in$ $R^{3 \times 3}$ are symmetric and positive definite inertia matrixes, $V_{m j}\left(q_{j}, \dot{q}_{j}\right) \in R^{3 \times 3}$ are the centripetal and coriolis matrixes, $G_{j}\left(q_{j}\right)=0 \in R^{3}$ are the gravitational vectors, $B_{j}\left(q_{j}\right) \in R^{3 \times 2}$ are the input transformation matrixes, $\tau_{j} \in R^{2}$ are the input torque vectors, $A_{j}\left(q_{j}\right) \in R^{1 \times 3}$ are the vectors associated with the constraints, and $\lambda_{j} \in R$ are the values of constraint forces. The nonholonomic constraints of the mobile robots are given by

$$
\dot{y}_{j} \cos \theta_{j}-\dot{x}_{j} \sin \theta_{j}-d_{j} \dot{\theta}=0,
$$

which can be written as

$$
A_{j}\left(q_{j}\right) \dot{q}_{j}=0
$$

with $A_{j}\left(q_{j}\right)=\left[\begin{array}{lll}-\sin \theta_{j} & \cos \theta_{j} & -d_{j}\end{array}\right]$.
If $S_{j}\left(q_{j}\right)$ and $V_{j}(t)$ are chosen as

$$
S_{j}\left(q_{j}\right)=\left[\begin{array}{cc}
\cos \theta_{j} & -d_{j} \sin \theta_{j} \\
\sin \theta_{j} & d_{j} \cos \theta_{j} \\
0 & 1
\end{array}\right], \quad V_{j}=\left[\begin{array}{c}
v_{j} \\
\omega_{j}
\end{array}\right],
$$

where $v_{j}, \omega_{j}$ are the linear and angular velocity of the robot respectively, then the dynamics (1) can be rewritten as

$$
\begin{gathered}
\dot{q}_{j}=S_{j}\left(q_{j}\right) V_{j}, \\
\bar{M}_{j} \dot{V}_{j}+\bar{V}_{m j} V_{j}=\bar{\tau}_{j},
\end{gathered}
$$

where $\bar{M}_{j}=S_{j}^{T} M_{j} S_{j}, \bar{V}_{m j}=S_{j}^{T}\left(M_{j} \dot{S}_{j}+V_{m j} S_{j}\right)$, and $\bar{\tau}_{j}=S_{j}^{T} B_{j} \tau_{j}$. There are three properties for the new set of coordinates [22].

Property 1. $\bar{M}_{j}$ is a symmetric positive definite matrix.

Property 2. $\left\|\bar{M}_{j}\right\|_{F} \leq \delta_{j},\left\|\bar{V}_{m j}\right\|_{F} \leq \rho_{j}\left\|\dot{q}_{j}\right\|$, where $\|\cdot\|_{F}$ denotes Frobenius norm of matrix and $\|\cdot\|$ denotes Euclidean norm of vector.

Property 3. The matrix $\dot{\bar{M}}_{j}-2 \bar{V}_{m j}$ is skew symmetric.

2.2. RBF Neural Networks. In adaptive control, NNs and fussy systems are widely used to approximate the unknown nonlinearities due to their inherent approximation capabilities [2227]. The universal approximation property of NNs [28] results in that, for any continuous function $f(X): \Omega_{X} \rightarrow R^{3}$, there exists a NN such that

$$
f(X)=W^{T} \phi(X)+\epsilon(X),
$$

where $\Omega_{X} \subset R^{m}$ is a compact set, $X \in \Omega_{X}$ is the input vector, $W \in R^{n \times 2}$ is the ideal weight matrix, $n>1$ is the number of the neurons, $\epsilon(X)$ is the ideal approximation error, $\phi(X)=$ $\left[\phi_{1}(X), \phi_{2}(X), \ldots, \phi_{n}(X)\right]^{T} \in R^{n}$ with $\phi_{i}(\cdot) \in R$ being the RBF functions, and $\phi_{i}(\cdot)$ are given by

$$
\phi_{i}(X)=\exp \left(-\frac{\left\|X-\xi_{i}\right\|^{2}}{2 b_{i}^{2}}\right), \quad i=1,2, \ldots, n
$$

in which $\xi_{i} \in R^{q}, b_{i} \in R$ are the center and width of the $i$ th neuron, respectively. The reconstruction error $\epsilon(x)$ is bounded on a compact set $S$ by $\|\epsilon(X)\| \leq \epsilon_{N}$. Moreover, for any $\epsilon_{N}$ one can find an NN such that $\|\epsilon(X)\| \leq \epsilon_{N}$ for all $X \in S$. The approximation weights $W$ are ideal target weights, and it is assumed that they are bounded such that $\|W\|_{F} \leq W_{M}$.

\subsection{Some Results on Finite Time Control}

Lemma 1 (see [29]). Consider the following system: $\dot{x}=f(x)$, $f(0)=0, x \in R^{n}$. Suppose there exists a positive definite continuous function $V(x): U \rightarrow R$, real number $c>0$ and $\gamma \in(0,1)$, and an open neighborhood $U_{0} \subset U$ of the origin such that $\dot{V}(x)+c(V(x))^{\gamma} \leq 0, x \in U_{0} \backslash\{0\}$. Then $V(x)$ approaches zero in finite time. In addition, the finite settling time $T$ satisfies that $T \leq V(x(0))^{1-\gamma} /[c(1-\gamma)]$. 
To facilitate the following lemma and the subsequent control design, define the vector $\operatorname{Sig}(\cdot)^{\gamma} \in R^{n}$ as follows:

$$
\operatorname{Sig}(\xi)^{\gamma}=\left[\left|\xi_{1}\right|^{\gamma} \operatorname{sgn}\left(\xi_{1}\right), \ldots,\left|\xi_{n}\right|^{\gamma} \operatorname{sgn}\left(\xi_{n}\right)\right]^{T},
$$

where $\xi=\left[\xi_{1}, \ldots, \xi_{n}\right]^{T} \in R^{n}, 0<\gamma<1$, and $\operatorname{sgn}(\cdot)$ is the standard signum function.

Lemma 2 (see $[30,31]$ ). If the vector $\xi \in R^{n}$ satisfies $\ddot{\xi}+$ $K \operatorname{Sig}(\xi)^{\alpha_{1}}+\bar{K} \operatorname{Sig}(\dot{\xi})^{\alpha_{2}}=0$ with positive constants $K, \bar{K}$ and $0<\alpha_{1}, \alpha_{2}<1$, then $\xi$ and $\dot{\xi}$ converge to zero in finite time.

\section{Leader-Follower Tracking}

In this section, control laws will be designed for the follower robot so that the leader and follower can track the desired trajectory in certain formation in finite time. The parameters of the desired trajectory are $q_{d}, V_{d}$, and $\dot{V}_{d}$. Define a reference position $X_{f r}=\left(x_{f r}, y_{f r}\right)^{T}$ for the follower robot:

$$
\begin{aligned}
X_{f r} & =\left[\begin{array}{l}
x_{f r} \\
y_{f r}
\end{array}\right] \\
& =\left[\begin{array}{l}
x_{l} \\
y_{l}
\end{array}\right]+\left[\begin{array}{l}
-d_{l} \cos \theta_{l}+L_{d} \cos \left(\Psi_{d}+\theta_{l}\right) \\
-d_{l} \sin \theta_{l}+L_{d} \sin \left(\Psi_{d}+\theta_{l}\right)
\end{array}\right],
\end{aligned}
$$

where $L_{d}$ and $\Psi_{d}$ are the desired separation and bearing, respectively. Taking first and second derivatives of $X_{f r}$, one gets

$$
\begin{aligned}
\dot{X}_{f r}= & {\left[\begin{array}{c}
\dot{x}_{l} \\
\dot{y}_{l}
\end{array}\right]+\left[\begin{array}{c}
d_{l} \sin \theta_{l}-L_{d} \sin \left(\Psi_{d}+\theta_{l}\right) \\
-d_{l} \cos \theta_{l}+L_{d} \cos \left(\Psi_{d}+\theta_{l}\right)
\end{array}\right] \omega_{l} } \\
\ddot{X}_{f r}= & {\left[\begin{array}{c}
\ddot{x}_{l} \\
\ddot{y}_{l}
\end{array}\right]+\left[\begin{array}{c}
d_{l} \sin \theta_{l}-L_{d} \sin \left(\Psi_{d}+\theta_{l}\right) \\
-d_{l} \cos \theta_{l}+L_{d} \cos \left(\Psi_{d}+\theta_{l}\right)
\end{array}\right] \dot{\omega}_{l} } \\
& +\left[\begin{array}{c}
d_{l} \cos \theta_{l}-L_{d} \cos \left(\Psi_{d}+\theta_{l}\right) \\
d_{l} \sin \theta_{l}-L_{d} \sin \left(\Psi_{d}+\theta_{l}\right)
\end{array}\right] \omega_{l}^{2} .
\end{aligned}
$$

From Figure 1, the current position of the follower robot can be stated as

$$
X_{f}=\left[\begin{array}{l}
x_{f} \\
y_{f}
\end{array}\right]=\left[\begin{array}{l}
x_{l} \\
y_{l}
\end{array}\right]+\left[\begin{array}{l}
-d_{l} \cos \theta_{l}+L \cos \left(\Psi+\theta_{l}\right) \\
-d_{l} \sin \theta_{l}+L \sin \left(\Psi+\theta_{l}\right)
\end{array}\right],
$$

where $L$ and $\Psi$ are the current separation and bearing angle, respectively.

If the follower's position $X_{f}$ and the reference position $X_{f r}$ satisfy $\lim _{t \rightarrow \infty}\left(X_{f}-X_{f r}\right)=0$, from (10) and (12), we can conclude that $\lim _{t \rightarrow \infty}\left(L-L_{d}\right)=0$ and $\lim _{t \rightarrow \infty}\left(\Psi-\Psi_{d}\right)=0$. In the following part of this paper, we try to design control law for the follower robot to track the reference position $X_{f r}$, so as to track the leader robot in the desired separation and bearing. We assume that only the pose $q_{l}=\left[x_{l}, y_{l}, \theta_{l}\right]^{T}$ of the leader robot can be obtained by the follower robot. The velocity and the system dynamics of the leader robot are unknown to the follower. From (5) and (6) we know that to design control law for the follower to track the reference position $X_{f r}$, the $\dot{X}_{l}$ and $\ddot{X}_{l}$ are needed. So some observers are needed to estimate the $\dot{X}_{l}$ and $\ddot{X}_{l}$.

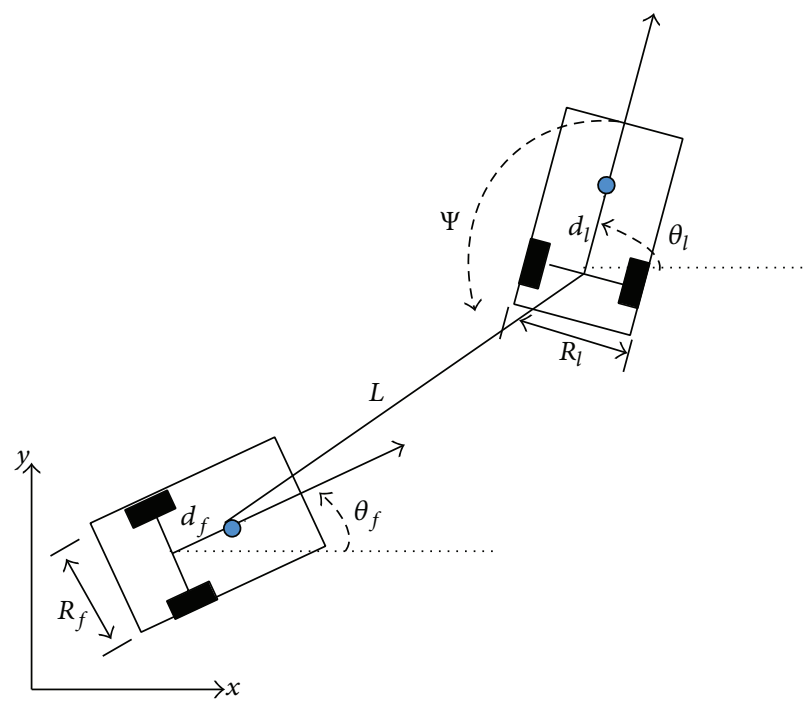

FIGURE 1: Leader-follower formation.

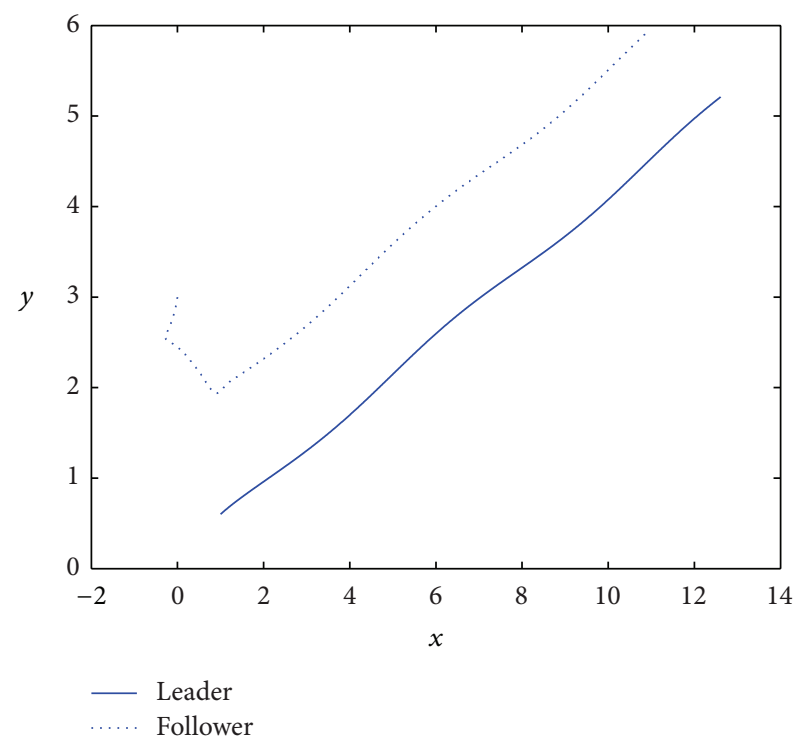

FIgURE 2: Trajectories of the leader and the follower.

3.1. NN Finite-Time Observer. In our previous paper [22], an $\mathrm{NN}$ observer is designed for the follower robot to estimate $\dot{X}_{l}$ and $\ddot{X}_{l}$. Here, an NN finite-time observer is designed to estimate $\dot{X}_{l}$ and $\ddot{X}_{l}$ by the use of the pose of the leader. Let $p_{l}=$ $S\left(q_{l}\right) V_{l}$; then, according to the leader's dynamics described in (5) and (6) and $\bar{\tau}_{l}=\bar{\tau}_{l}\left(q_{l}, p_{l}, q_{d}, V_{d}, \dot{V}_{d}\right)$, the dynamics of the leader robot can be restated as

$$
\begin{gathered}
\dot{q}_{l}=p_{l}, \\
\dot{p}_{l}=f_{l}\left(q_{l}, p_{l}, q_{d}, V_{d}, \dot{V}_{d}\right),
\end{gathered}
$$

where $f_{l}\left(q_{l}, p_{l}, q_{d}, V_{d}, \dot{V}_{d}\right)=\dot{S}\left(q_{l}\right) V_{l}-S\left(q_{l}\right) \bar{M}_{l}^{-1}\left(\bar{V}_{m l} V_{l}-\bar{\tau}_{l}\right)$. 


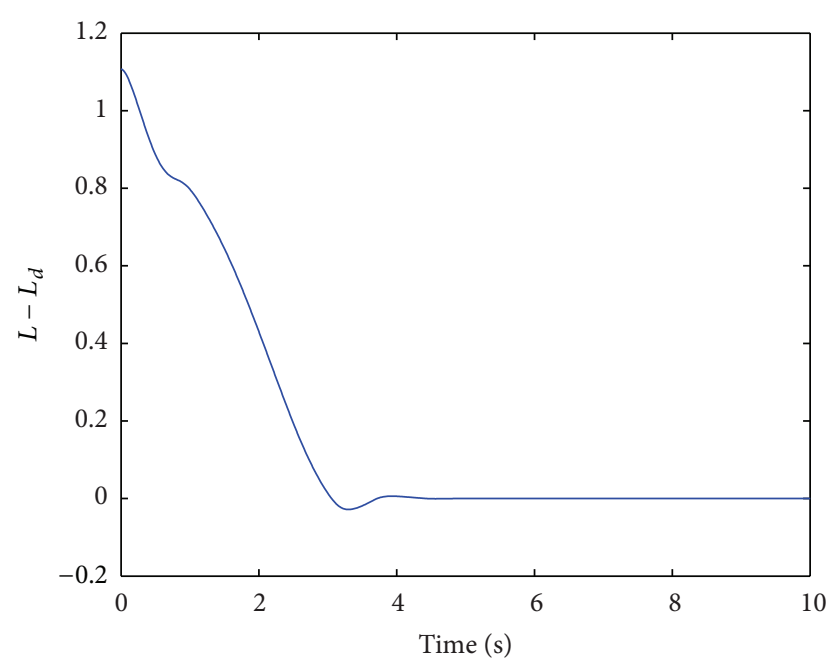

FIGURE 3: Separation tracking errors $L-L_{d}$.

It is convenient to design the following $\mathrm{NN}$ finite-time observer:

$$
\begin{gathered}
\dot{\hat{q}}_{l}=\widehat{p}_{l}+k_{1} \operatorname{sgn}\left(\widetilde{q}_{l}\right)+K_{1} \widetilde{q}_{l}^{\alpha}, \\
\dot{\hat{p}}_{l}=\widehat{f}_{l}\left(q_{l}, \widehat{p}_{l}, q_{d}, V_{d}, \dot{V}_{d}\right)+k_{2} \operatorname{sgn}\left(u_{l}\right)+K_{2} u_{l}^{\beta},
\end{gathered}
$$

where $\widehat{f}_{l}\left(q_{l}, \widehat{p}_{l}, q_{d}, V_{d}, \dot{V}_{d}\right)=\widehat{W}^{T} \widehat{\phi}=\widehat{W}^{T} \phi\left(q_{l}, \widehat{p}_{l}, q_{d}, V_{d}, \dot{V}_{d}\right)$ is the NN estimation of the function $f_{l}\left(q_{l}, p_{l}, q_{d}, V_{d}, \dot{V}_{d}\right)=$ $W^{T} \phi+\epsilon=W^{T} \phi\left(q_{l}, p_{l}, q_{d}, V_{d}, \dot{V}_{d}\right)+\epsilon ; \widetilde{q}, \widetilde{p}_{l}$ as the estimation errors are defined as $\widetilde{q}_{l}=q_{l}-\widehat{q}_{l}$ and $\widetilde{p}_{l}=p_{l}-\widehat{p}_{l} ; 0<\alpha=l_{\alpha} / s_{\alpha}$, $\beta=l_{\beta} / s_{\beta}<1$ with $l_{\alpha}, l_{\beta}, s_{\alpha}$ and $s_{\beta}$ are some positive odd integers; $u_{l}=k_{1} \operatorname{sgn}\left(\widetilde{q}_{l}\right)+K_{1} \widetilde{q}_{l}^{\alpha} ; k_{1} \geq|\widetilde{p}|, K_{1}, k_{2}$, and $K_{2}$ are some designed positive constants. Then the time derivative of $\tilde{q}_{l}$ and $\tilde{p}_{l}$ can be written as

$$
\begin{gathered}
\dot{\tilde{q}}_{l}=\widetilde{p}_{l}-k_{1} \operatorname{sgn}\left(\widetilde{q}_{l}\right)-K_{1} \widetilde{q}_{l}^{\alpha}, \\
\dot{\tilde{p}}_{l}=f_{l}-\widehat{f}_{l}-k_{2} \operatorname{sgn}\left(u_{l}\right)-K_{2} u_{l}^{\beta} \\
=W^{T} \phi+\epsilon-\widehat{W}^{T} \widehat{\phi}-k_{2} \operatorname{sgn}\left(u_{l}\right)-K_{2} u_{l}^{\beta} \\
=W^{T} \phi-W^{T} \widehat{\phi}+W^{T} \widehat{\phi}-\widehat{W}^{T} \widehat{\phi}+\epsilon \\
-k_{2} \operatorname{sgn}\left(u_{l}\right)-K_{2} u_{l}^{\beta} \\
=W^{T} \tilde{\phi}+\widetilde{W}^{T} \hat{\phi}+\epsilon-k_{2} \operatorname{sgn}\left(u_{l}\right)-K_{2} u_{l}^{\beta},
\end{gathered}
$$

where $\widetilde{W}=W-\widehat{W}, \widetilde{\phi}=\phi-\widehat{\phi}$, and $k_{2}$ is a positive constant to be designed.

Taking time derivative of the Lyapunov function $V_{1}=$ $(1 / 2) \widetilde{q}_{l}^{2}$ and substituting (15), one gets

$$
\dot{V}_{1}=\widetilde{q}_{l}\left(\tilde{p}_{l}-k_{1} \operatorname{sgn}\left(\widetilde{q}_{l}\right)-K_{1} \widetilde{q}_{l}^{\alpha}\right) \leq-2 K_{1} V_{1}^{(\alpha+1) / 2} .
$$

From (17) and $0<(\alpha+1) / 2<1$, we can conclude that the estimation error $\widetilde{q}_{l}$ converges to zero in finite time. Since $W, \widetilde{\phi}$ and the approximation error $\epsilon$ in (16) are bounded,

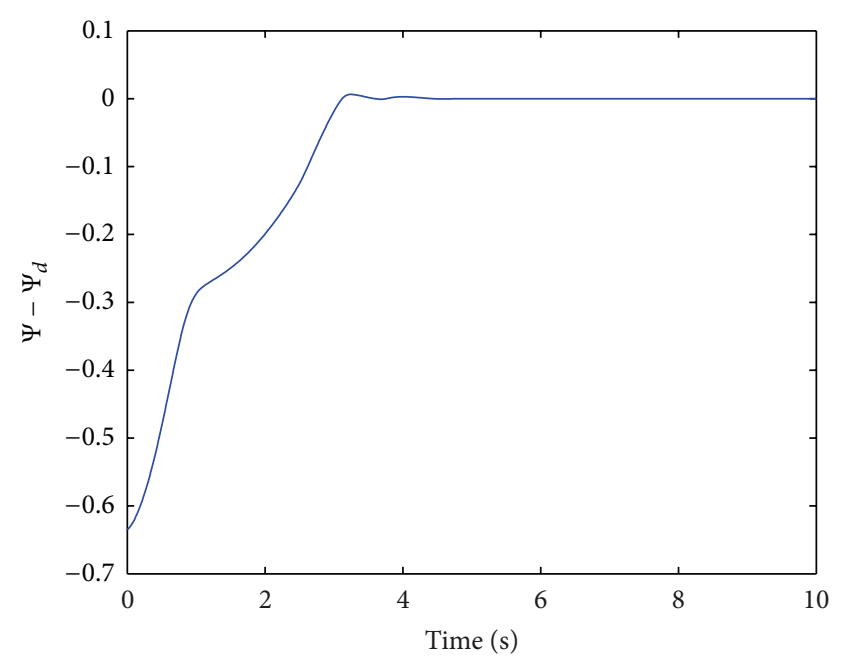

FIGURE 4: Bearing angle tracking errors $\Psi-\Psi_{d}$.

respectively, there exists a positive constant $\zeta_{0}$ such that $\left|W^{T} \widetilde{\phi}+\epsilon\right| \leq \zeta_{0}$.

Taking time derivative of the Lyapunov function $V_{2}=$ $(1 / 2) \widetilde{p}_{l}^{T} \widetilde{p}_{l}+(1 / 2) \widetilde{W}^{T} F^{-1} \widetilde{W}$ with $F$ a positive definite constant matrix and substituting (16), one obtains

$$
\begin{aligned}
\dot{V}_{2}= & \widetilde{p}_{l}^{T}\left(W^{T} \tilde{\phi}+\epsilon-k_{2} \operatorname{sgn}\left(u_{l}\right)-K_{2} u_{l}^{\beta}\right) \\
& +\widetilde{W}^{T}\left(F^{-1} \dot{\widetilde{W}}+\widehat{\phi} \widetilde{p}_{l}^{T}\right) .
\end{aligned}
$$

From (15), on the sliding surface $\dot{\tilde{q}}_{l}=0$, one has $u_{l}=\widetilde{p}_{l}$. If $k_{2}>\zeta_{0}$ and the weight $\widehat{W}$ is updated by

$$
\dot{\widetilde{W}}=-\dot{\widehat{W}}=-F \hat{\phi} u_{l}^{T},
$$

then

$$
\dot{V}_{2} \leq-K_{2}\left\|\tilde{p}_{l}\right\|^{1+\beta}
$$

from which we know that $\widetilde{W}$ are bounded. From the boundedness of $\widetilde{W}, \widehat{\phi}$ and the inequality $\left\|\widetilde{W}^{T} \widehat{\phi}\right\|_{F} \leq\|\widetilde{W}\|_{F}\|\widehat{\phi}\|$, it can be concluded that $\left\|\widetilde{W}^{T} \widehat{\phi}\right\|_{F}$ is bounded.

Taking time derivative of $V_{3}=(1 / 2) \tilde{p}_{l}^{T} \tilde{p}_{l}$ and substituting $u_{l}=\widetilde{p}_{l}$, one gets

$$
\dot{V}_{3}=\widetilde{p}_{l}^{T}\left(W^{T} \widetilde{\phi}+\widetilde{W}^{T} \hat{\phi}+\epsilon-k_{2} \operatorname{sgn}\left(\widetilde{p}_{l}\right)-K_{2} \tilde{p}_{l}^{\beta}\right) .
$$

If the gain $k_{2}$ is designed such that $k_{2} \geq \zeta_{0}+\|\widetilde{W}\|_{F}\|\widehat{\phi}\|$, then

$$
\dot{V}_{3} \leq-K_{2} \tilde{p}_{l}^{T} \tilde{p}_{l}^{\beta}=-2 K_{2} V_{3}^{(\beta+1) / 2} .
$$

From (22) and $0<(\beta+1) / 2<1$, it is obtained that the estimation error $\tilde{p}_{l}$ converges to zero in finite time. Consequently, the following theorem can be established by the above analysis directly. 

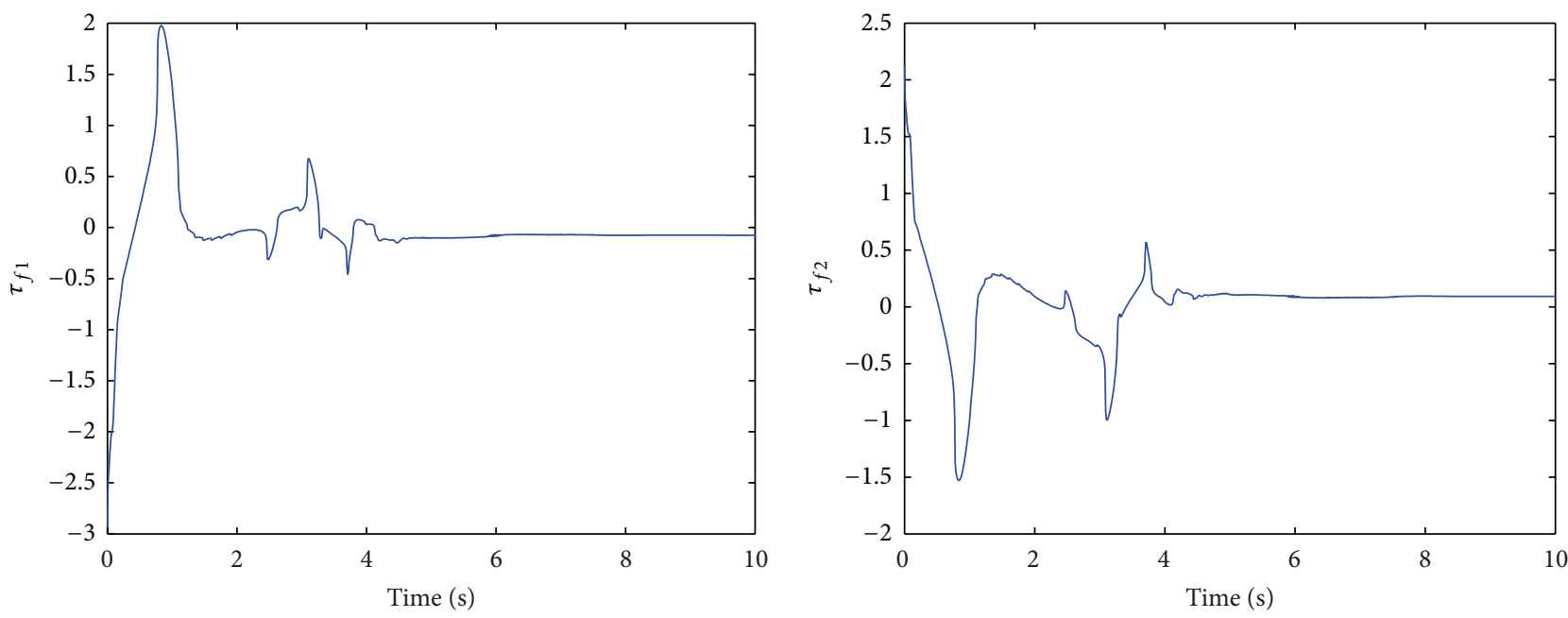

FIGURE 5: The control law $\tau_{f}=\left[\tau_{f 1}, \tau_{f 2}\right]^{T}$ for the follower robot.

Theorem 3. Suppose only the leader robot's position $q_{l}$ is possible to the follower robot. If the finite time observer described in (15) and (16) is used to estimate the leader's dynamics and $k_{2} \geq \zeta_{0}+\|\widetilde{W}\|_{F}\|\widehat{\phi}\|$, then the estimation errors $\widetilde{q}_{l}, \widetilde{p}_{l}$ converge to zero in finite time.

Remark 4. The observer design in this part is to estimate the $\dot{X}_{f r}$ and $\ddot{X}_{f r}$. Since $\widehat{q}_{l}, \widehat{p}_{l}$ are the estimates of $q_{l}, p_{l}$, from (11) the estimation of $\dot{X}_{f r}$ and $\ddot{X}_{f r}$ can be stated as follows:

$$
\begin{aligned}
\widehat{\dot{X}}_{f r}= & {\left[\begin{array}{lll}
1 & 0 & 0 \\
0 & 1 & 0
\end{array}\right] \widehat{p}_{l} } \\
& +\left[\begin{array}{c}
d_{l} \sin \theta_{l}-L_{d} \sin \left(\Psi_{d}+\theta_{l}\right) \\
-d_{l} \cos \theta_{l}+L_{d} \cos \left(\Psi_{d}+\theta_{l}\right)
\end{array}\right] \hat{\dot{\theta}}_{l}, \\
\widehat{\ddot{X}}_{f r}= & \frac{d \widehat{\dot{X}}_{f r}}{d t} \\
= & {\left[\begin{array}{lll}
1 & 0 & 0 \\
0 & 1 & 0
\end{array}\right] \dot{\hat{p}}_{l} } \\
& +\left[\begin{array}{c}
d_{l} \sin \theta_{l}-L_{d} \sin \left(\Psi_{d}+\theta_{l}\right) \\
-d_{l} \cos \theta_{l}+L_{d} \cos \left(\Psi_{d}+\theta_{l}\right)
\end{array}\right]\left[\begin{array}{lll}
0 & 0 & 1
\end{array}\right] \dot{\vec{p}}_{l} \\
& +\left[\begin{array}{l}
d_{l} \cos \theta_{l}-L_{d} \cos \left(\Psi_{d}+\theta_{l}\right) \\
d_{l} \sin \theta_{l}-L_{d} \sin \left(\Psi_{d}+\theta_{l}\right)
\end{array}\right] \hat{\dot{\theta}}_{l}^{2} .
\end{aligned}
$$

Furthermore, from (10) and (21), we can get the following estimation error:

$$
\begin{aligned}
\widetilde{\dot{X}}_{f r}= & \dot{X}_{f r}-\widehat{\dot{X}}_{f r} \\
= & {\left[\begin{array}{lll}
1 & 0 & 0 \\
0 & 1 & 0
\end{array}\right] \tilde{p}_{l} } \\
& +\left[\begin{array}{c}
d_{l} \sin \theta_{l}-L_{d} \sin \left(\Psi_{d}+\theta_{l}\right) \\
-d_{l} \cos \theta_{l}+L_{d} \cos \left(\Psi_{d}+\theta_{l}\right)
\end{array}\right]\left[\begin{array}{lll}
0 & 0 & 1
\end{array}\right] \widetilde{p}_{l} \\
= & {\left[\begin{array}{ccc}
1 & 0 & d_{l} \sin \theta_{l}-L_{d} \sin \left(\Psi_{d}+\theta_{l}\right) \\
0 & 1 & -d_{l} \cos \theta_{l}+L_{d} \cos \left(\Psi_{d}+\theta_{l}\right)
\end{array}\right] \widetilde{p}_{l} . }
\end{aligned}
$$

Remark 5. From Theorem 3 and the expressions (23) and (24), we can conclude that the estimation errors $\widetilde{\dot{X}}_{f r}$ and $\widetilde{\ddot{X}}_{f r}$ converge to zero in finite time.

3.2. Leader-Following Formation Control. In this part, a finite-time control law will be designed for the follower robot to track the leader robot in the desired separation and bearing. Define the leader-following formation tracking error as

$$
e_{f}=X_{f}-X_{f r}
$$

and denote the matrices $Q, N$ as

$$
\begin{gathered}
Q=\left[\begin{array}{cc}
\cos \theta_{f} & -d_{f} \sin \theta_{f} \\
\sin \theta_{f} & d_{f} \cos \theta_{f}
\end{array}\right], \\
N=Q^{-1}=\left[\begin{array}{cc}
\cos \theta_{f} & \sin \theta_{f} \\
\frac{-\sin \theta_{f}}{d_{f}} & \frac{\cos \theta_{f}}{d_{f}}
\end{array}\right] .
\end{gathered}
$$

From the follower's dynamics described in (5), it is easy to know that $\left[\begin{array}{ll}\dot{x}_{f} & \dot{y}_{f}\end{array}\right]^{T}=Q V_{f}$; that is, $\dot{X}_{f}=Q V_{f}$. Then differentiating both sides of (25) yields

$$
\begin{gathered}
\dot{e}_{f}=\dot{X}_{f}-\dot{X}_{f r}=Q V_{f}-\dot{X}_{f r}, \\
\ddot{e}_{f}=Q \bar{M}_{f}^{-1}\left(-\bar{V}_{m} V_{f}+\bar{\tau}_{f}\right)+\dot{Q} V_{f} \ddot{X}_{f r} .
\end{gathered}
$$

If the control law $\bar{\tau}_{f}$ for the following robot is designed such that

$$
\begin{aligned}
Q \bar{M}_{f}^{-1} \bar{\tau}_{f}= & Q \bar{M}_{f}^{-1} \bar{V}_{m f} V_{f}-\dot{Q} V_{f}+\widehat{\ddot{X}}_{f r} \\
& -k_{f 1} \operatorname{sig}\left(e_{f}\right)^{\alpha_{1}}-k_{f 2} \operatorname{sig}\left(\dot{e}_{f}\right)^{\alpha_{2}}
\end{aligned}
$$

with $0<\alpha_{1}, \alpha_{2}<1, \alpha_{2}=2 \alpha_{1} /\left(\alpha_{1}+1\right)$, and $k_{f 1}, k_{f 2}$ being some designed positive gains, then

$$
\ddot{e}_{f}=-k_{f 1} \operatorname{sig}\left(e_{f}\right)^{\alpha_{1}}-k_{f 2} \operatorname{sig}\left(\dot{e}_{f}\right)^{\alpha_{2}}-\widetilde{\dot{X}}_{f r} .
$$




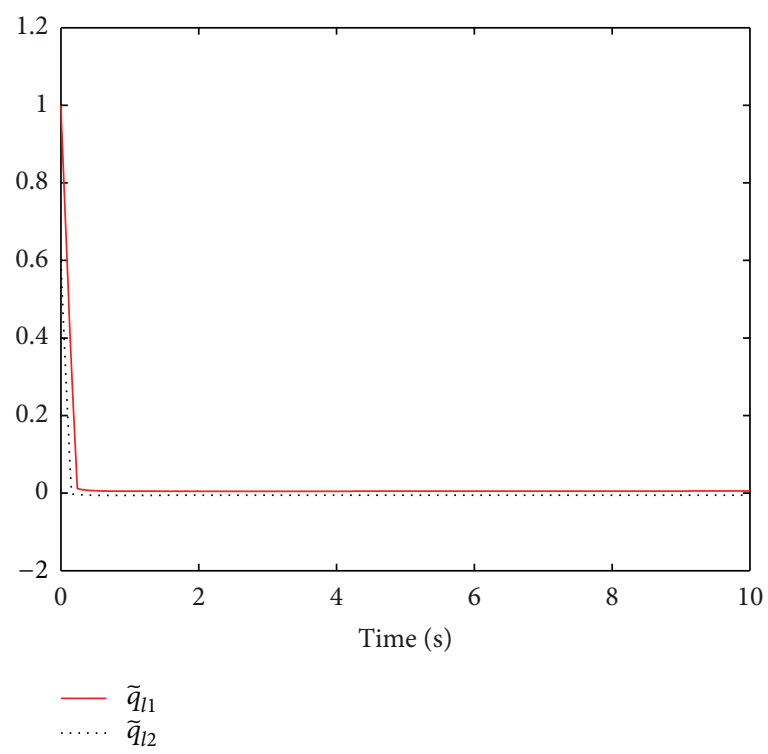

(a) The finite-time observer estimation error $\widetilde{q}_{l 1}$

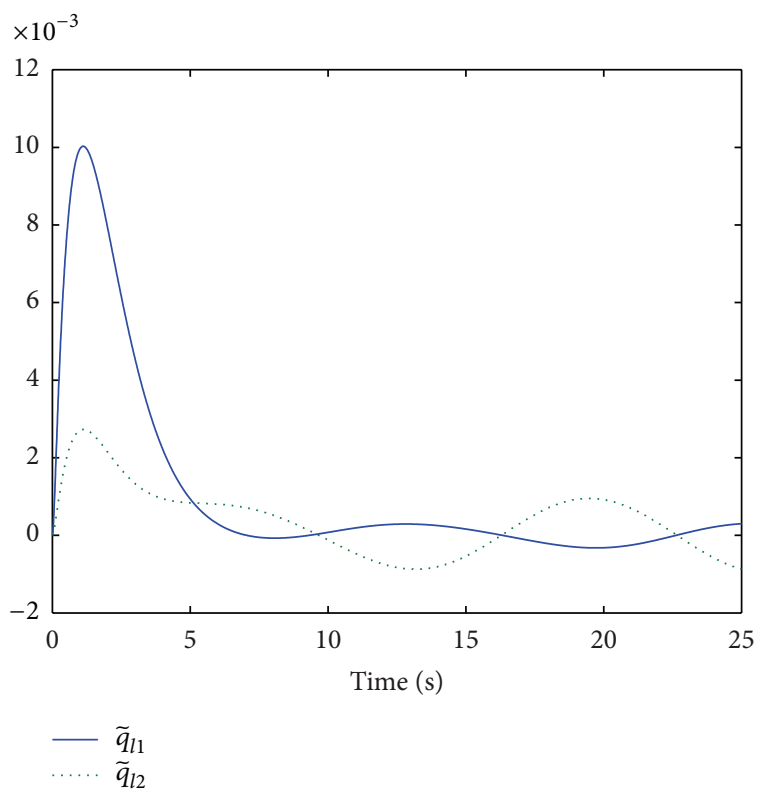

(c) The infinite-time observer estimation error $\widetilde{q}_{l 1}$

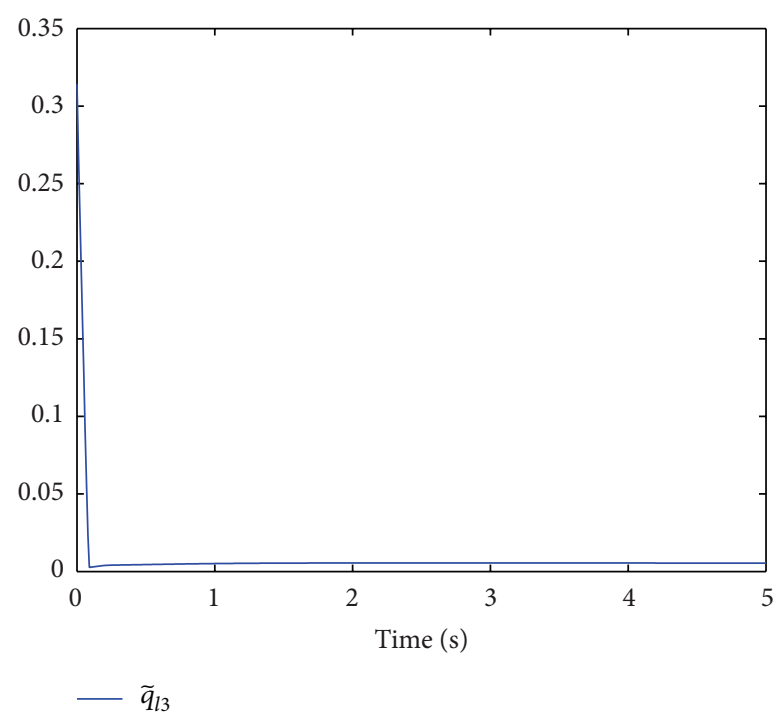

(b) The finite-time observer estimation error $\widetilde{q}_{l 2}, \widetilde{q}_{l 3}$

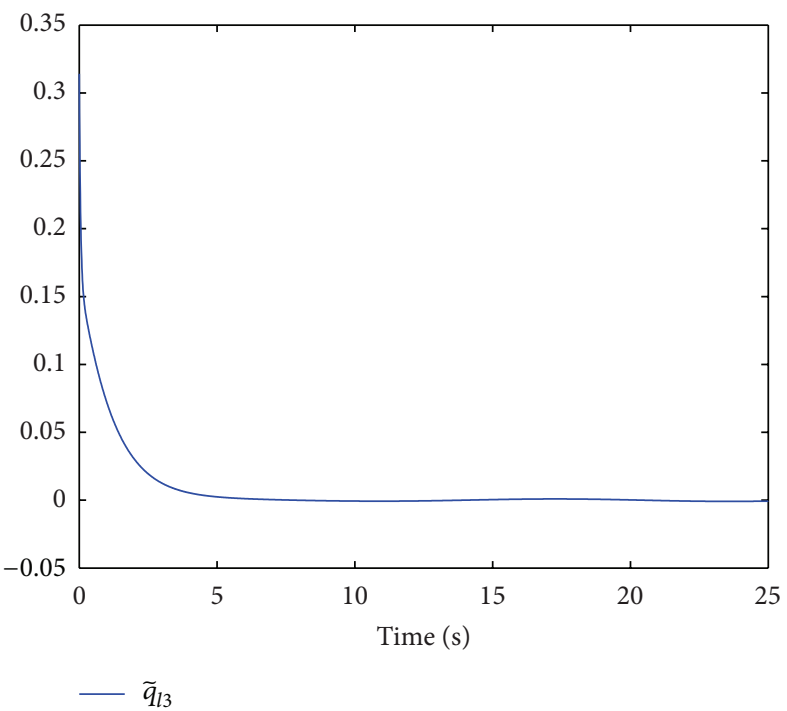

(d) The infinite-time observer estimation error $\tilde{q}_{l 2}, \widetilde{q}_{l 3}$

FIGURE 6: The infinite-time observer and finite-time observer estimation errors.

The following theorem illustrates the efficiency of the finitetime control law $\bar{\tau}_{f}$ for the following robot. The proof is omitted since it can be directly obtained by the finite-time convergence of $\widetilde{\dot{X}}_{f r}$ and Lemma 2 .

Theorem 6. Consider the leader robot and the following robot with the dynamics described by (5) and (6). If NN finite-time observer by the following robot is designed as (14) with the $\mathrm{NN}$ weight updated law defined by (19) and the control law $\bar{\tau}_{f}$ for the following robot described by (28), then the leader-following tracking error $e_{f}$ and its time derivative $\dot{e}_{f}$ converge to zero in finite time.

\section{Simulation Results}

The dynamics of the nonholonomic mobile robots can be derived using Lagrangian methods [22] and written in the form (1), where

$$
\begin{gathered}
M_{j}\left(q_{j}\right)=\left[\begin{array}{ccc}
m_{j} & 0 & m_{j} d_{j} \sin \theta_{j} \\
0 & m_{j} & -m_{j} d_{j} \cos \theta_{j} \\
m_{j} d_{j} \sin \theta_{j} & -m_{j} d_{j} \cos \theta_{j} & I_{j}
\end{array}\right], \\
B_{j}\left(q_{j}\right)=\frac{1}{r_{j}}\left[\begin{array}{cc}
\cos \theta_{j} & \cos \theta_{j} \\
\sin \theta_{j} & \sin \theta_{j} \\
R_{j} & -R_{j}
\end{array}\right], \quad A_{j}^{T}\left(q_{j}\right)=\left[\begin{array}{c}
-\sin \theta_{j} \\
\cos \theta_{j} \\
-d_{j}
\end{array}\right],
\end{gathered}
$$




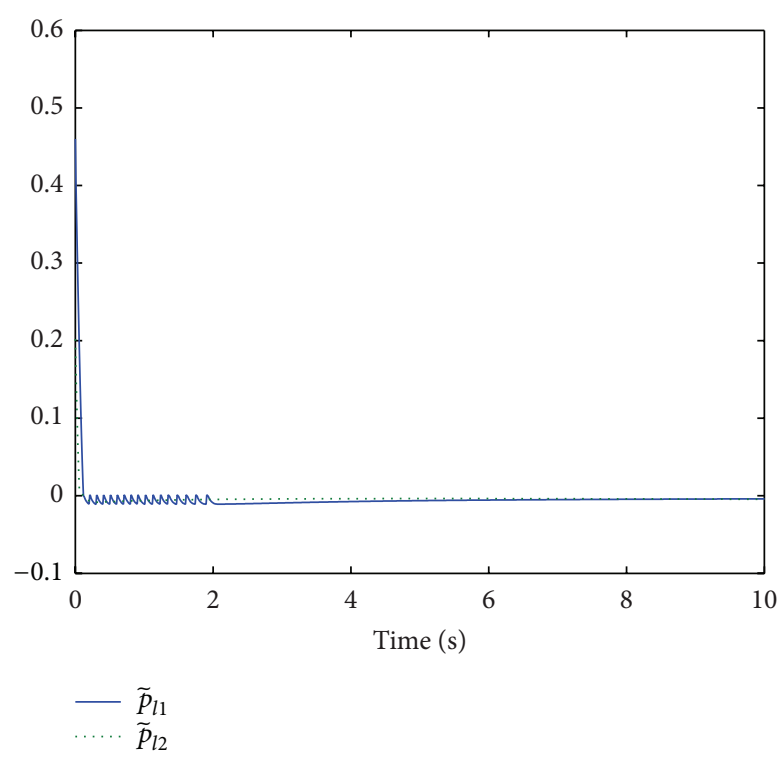

(a) The finite-time observer estimation error $\widetilde{p}_{l 1}, \widetilde{p}_{l 2}$

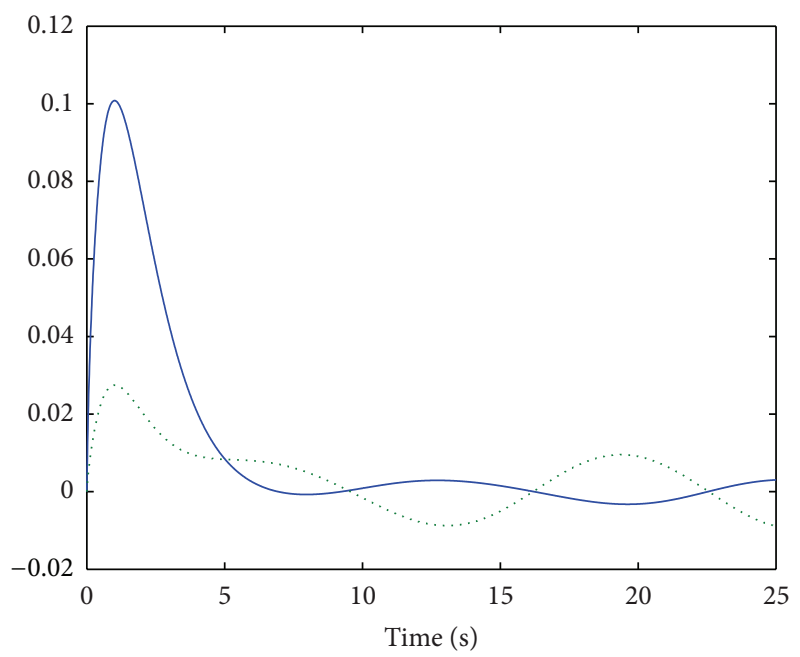

$-\tilde{p}_{l 1}$

(c) The infinite-time observer estimation error $\widetilde{p}_{l 1}, \widetilde{p}_{l 2}$

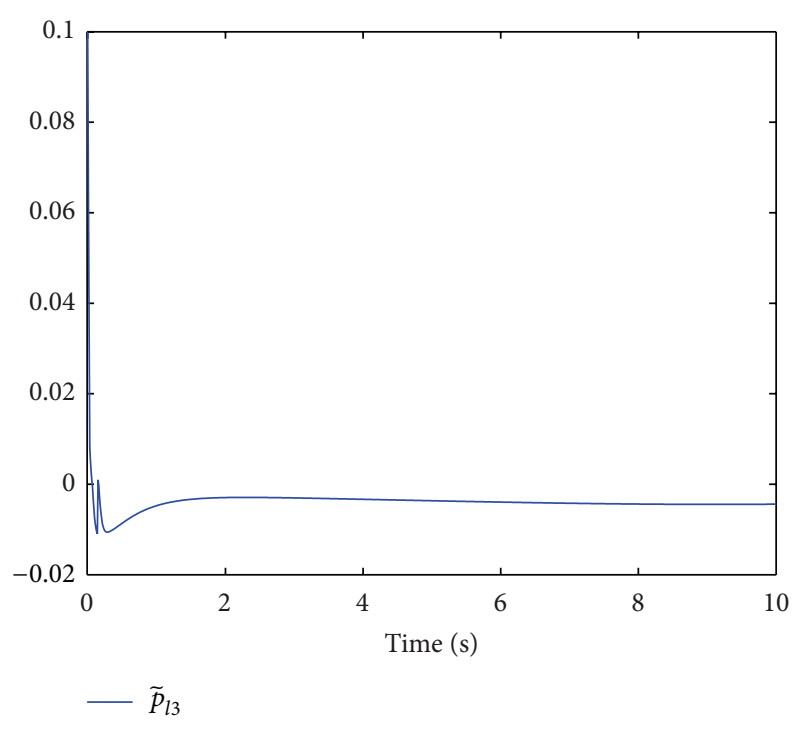

(b) The finite-time observer estimation error $\widetilde{q}_{13}$

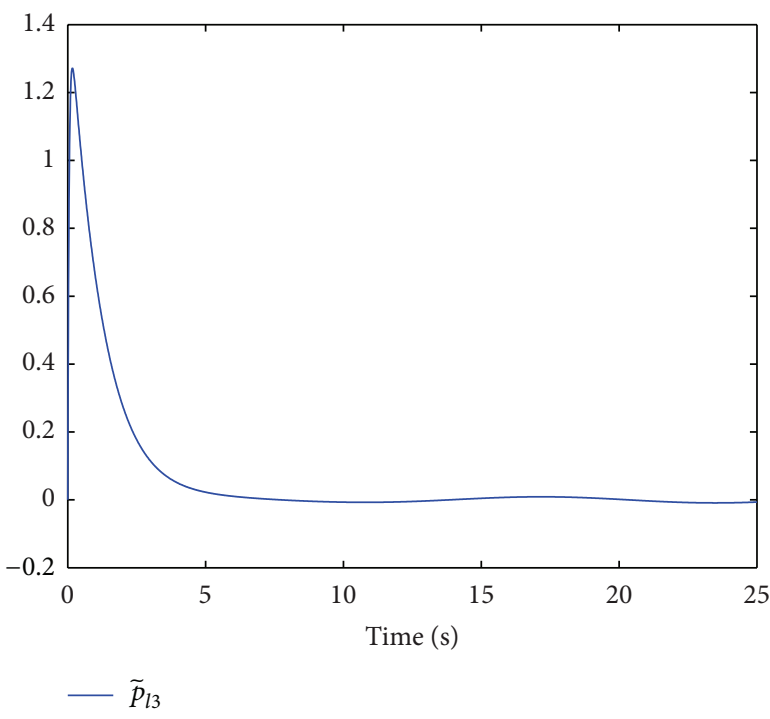

(d) The infinite-time observer estimation error $\widetilde{q}_{l 3}$

FIGURE 7: The infinite-time observer and finite-time observer estimation errors.

$$
\begin{aligned}
V_{m j}\left(q_{j}, \dot{q}_{j}\right) & =\left[\begin{array}{ccc}
0 & 0 & m_{j} d_{j} \dot{\theta}_{j} \cos \theta_{j} \\
0 & 0 & m_{j} d_{j} \dot{\theta}_{j} \sin \theta_{j} \\
0 & 0 & 0
\end{array}\right], \quad G\left(q_{j}\right)=0, \\
\lambda_{j} & =-m_{j}\left(\dot{x}_{j} \cos \theta_{j}+\dot{y}_{j} \sin \theta_{j}\right) \dot{\theta}_{j},
\end{aligned}
$$

where $j=l, f$ with $l, f$ denoting the leader robot and the follower robot, respectively. In this simulation, we take the parameters of the mobile robots as $m_{l}=m_{f}=10 \mathrm{~kg}, I_{l}=$ $I_{f}=5 \mathrm{~kg} \cdot \mathrm{m}^{2}, r_{l}=r_{f}=0.05, d_{l}=d_{f}=0.5, R_{l}=R_{f}=0.25$ and choose the initial conditions for the leader and follower as $q_{l}(0)=(1,0.6, \pi / 10)^{T}, V_{l}(0)=(0.5,0.1)^{T}, q_{f}=(0,3,0)^{T}$,
$V_{f}(0)=0$. Consider $V_{d}=\left[v_{d}, \omega_{d}\right]^{T}, v_{d}=0.5 \mathrm{~m} / \mathrm{s}, \omega_{d}=$ $-0.025 \cos (0.5 t) \mathrm{rad} / \mathrm{s}$ as the reference velocity for the leader robot and consider $L_{d}=1.5$ and $\Psi_{d}=2 \pi / 3$ as the desired separation and bearing between the leader and follower.

The simulation example is carried out on Lenovo G470 and Matlab 7.0 environments. Simulation results are shown in Figures 2-7. Figure 2 shows the trajectories of the leader and the follower. Figures 3 and 4 present the separation tracking errors $L-L_{d}$ and the bearing angles tracking errors $\Psi-\Psi_{d}$, respectively. From the two figures we can see that under the control law shown in Figure 5 the follower robot can well track the leader robot in desired separation $L_{d}$ and bearing $\Psi_{d}$ after 4 seconds. Figures 6 and 7 present the NN infinite-time observer in [22] and the NN finite-time observer estimation 
errors and $\tilde{q}_{l}$ and $\tilde{p}_{l}$. From the two figures we can see that the NN observer by the follower can estimate the dynamics of the leader robot with bounded errors and the estimation errors will be close to zero after 2 seconds. From the compare of the $\mathrm{NN}$ infinite-time observer and the NN finite-time observer estimation errors in Figures 6 and 7, we can know that the finite-time observer has higher convergence rates and higher estimation accuracy.

\section{Conclusions}

In this paper, a leader-following formation control has been designed for the following nonholonomic mobile robot to track the leading nonholonomic mobile robot in the desired separation and bearing angle in finite time. In the formation, the the dynamics of the leader robot is unknown to the follow and only the leader's pose (i.e., position and orientation angle) can be obtained by the follower. To design the control law for the following robot, the dynamics of the leader robot is needed. So an NN finite-time observer by the follower robot was designed to estimate the leader's dynamics. Based on the leader's estimated dynamics, a finite-time controller was designed for the follower robot to track the leader robot in the desired separation and bearing in finite time. The effectiveness of the proposed control design has been illustrated by formal proof and simulation results.

\section{Conflict of Interests}

The authors declare that there is no conflict of interests regarding the publication of this paper.

\section{Acknowledgments}

This work was supported by the National Natural Science Foundation of China (61304073), the Natural Science Foundation of Jiangsu Province (BK20130536, BK20130533), China Postdoctoral Science Foundation (2013M541615, 2013M540421), Postdoctoral Science Foundation of Jiangsu Province (1301044B), and Scientific Research Foundation for Advanced Talents by Jiangsu University.

\section{References}

[1] T. Balch and R. C. Arkin, "Behavior-based formation control for multirobot teams," IEEE Transactions on Robotics and Automation, vol. 14, no. 6, pp. 926-939, 1998.

[2] J. R. T. Lawton, R. W. Beard, and B. J. Young, "A decentralized approach to formation maneuvers," IEEE Transactions on Robotics and Automation, vol. 19, no. 6, pp. 933-941, 2003.

[3] M. Egerstedt and X. Hu, "Formation constrained multi-agent control," IEEE Transactions on Robotics and Automation, vol. 17, no. 6, pp. 947-951, 2001.

[4] S. J. Yoo, J. B. Park, and Y. H. Choi, "Adaptive formation tracking control of electrically driven multiple mobile robots," IET Control Theory and Applications, vol. 4, no. 8, pp. 1489-1500, 2010.

[5] W. J. Dong, Y. Guo, and J. A. Farrell, "Formation control of nonholonomic mobile robots," in Proceedings of the American
Control Conference, pp. 5602-5607, Minneapolis, Minn, USA, June 2006.

[6] W. Ren and N. Sorensen, "Distributed coordination architecture for multi-robot formation control," Robotics and Autonomous Systems, vol. 56, no. 4, pp. 324-333, 2008.

[7] H. G. Tanner, G. J. Pappas, and V. Kumar, "Leader-to-formation stability," IEEE Transactions on Robotics and Automation, vol. 20, no. 3, pp. 443-455, 2004.

[8] X. Li and J. Xiao, "Robot formation control in leader-follower motion using direct Lyapunov method," International Journal of Intelligent Control and Systems, vol. 10, pp. 244-250, 2005.

[9] J. Shao, G. Xie, and L. Wang, "Leader-following formation control of multiple mobile vehicles," IET Control Theory and Applications, vol. 1, no. 2, pp. 545-552, 2007.

[10] L. Consolini, F. Morbidi, D. Prattichizzo, and M. Tosques, "Leader-follower formation control of nonholonomic mobile robots with input constraints," Automatica, vol. 44, no. 5, pp. 1343-1349, 2008.

[11] T. Gustavi and X. Hu, "Observer-based leader-following formation control using onboard sensor information," IEEE Transactions on Robotics, vol. 24, no. 6, pp. 1457-1462, 2008.

[12] K. Choi, S. J. Yoo, J. B. Park, and Y. H. Choi, "Adaptive formation control in absence of leader's velocity information," IET Control Theory \& Applications, vol. 4, no. 4, pp. 521-528, 2010.

[13] J. Chen, D. Sun, J. Yang, and H. Chen, "Leader-follower formation control of multiple non-holonomic mobile robots incorporating a Receding-Horizon scheme," International Journal of Robotics Research, vol. 29, no. 6, pp. 727-747, 2010.

[14] T. Dierks and S. Jagannathan, "Asymptotic adaptive neural network tracking control of nonholonomic mobile robot formations," Journal of Intelligent and Robotic Systems, vol. 56, no. 1-2, pp. 153-176, 2009.

[15] T. Dierks and S. Jagannathan, "Neural network control of mobile robot formations using RISE feedback," IEEE Transactions on Systems, Man, and Cybernetics B: Cybernetics, vol. 39, no. 2, pp. 332-347, 2009.

[16] T. Dierks and S. Jagannathan, "Neural network output feedback control of robot formations," IEEE Transactions on Systems, Man, and Cybernetics B: Cybernetics, vol. 40, no. 2, pp. 383-399, 2010.

[17] B. S. Park, J. B. Park, and Y. H. Choi, "Robust adaptive formation control and collision avoidance for electrically driven nonholonomic mobile robots," IET Control Theory and Applications, vol. 5, no. 3, pp. 514-522, 2011.

[18] F. Xiao, L. Wang, J. Chen, and Y. Gao, "Finite-time formation control for multi-agent systems," Automatica, vol. 45, no. 11, pp. 2605-2611, 2009.

[19] Y. Cao, W. Ren, and Z. Meng, "Decentralized finite-time sliding mode estimators and their applications in decentralized finitetime formation tracking," Systems \& Control Letters, vol. 59, no. 9, pp. 522-529, 2010.

[20] S. Li, H. Du, and X. Lin, "Finite-time consensus algorithm for multi-agent systems with double-integrator dynamics," Automatica, vol. 47, no. 8, pp. 1706-1712, 2011.

[21] M. Ou, H. Du, and S. Li, "Finite-time formation control of multiple nonholonomic mobile robots," International Journal of Robust and Nonlinear Control, vol. 24, no. 1, pp. 140-165, 2014.

[22] T. Sun, F. Liu, H. Pei, and Y. He, "Observer-based adaptive leader-following formation control for non-holonomic mobile robots," IET Control Theory \& Applications, vol. 6, no. 18, pp. 2835-2841, 2012. 
[23] T. Sun, H. Pei, Y. Pan, H. Zhou, and C. Zhang, "Neural networkbased sliding mode adaptive control for robot manipulators," Neurocomputing, vol. 74, no. 14-15, pp. 2377-2384, 2011.

[24] T. Sun, H. Pei, Y. Pan, and C. Zhang, "Robust adaptive neutral network control for environmental boundary tracking by mobile robots," International Journal of Robust and Nonlinear Control, vol. 23, no. 2, pp. 123-136, 2013.

[25] Y. Pan, M. J. Er, D. Huang, and Q. Wang, "Adaptive fuzzy control with guaranteed convergence of optimal approximation error," IEEE Transactions on Fuzzy Systems, vol. 19, no. 5, pp. 807-818, 2011.

[26] Y. Pan and M. J. Er, "Enhanced adaptive fuzzy control with optimal approximation error convergence," IEEE Transactions on Fuzzy Systems, vol. 21, no. 6, pp. 1123-1132, 2013.

[27] Y. Pan, Y. Zhou, T. Sun, and M. J. Er, "Composite adaptive fuzzy $H^{\infty}$ tracking control of uncertain nonlinear systems," Neurocomputing, vol. 99, pp. 15-24, 2013.

[28] F. L. Lewis, S. Jagannathan, and A. Yesilderek, Neural Network Control of Robot Manipulators and Nonlinear Systems, Taylor \& Francis, London, UK, 1999.

[29] S. P. Bhat and D. S. Bernstein, "Finite-time stability of continuous autonomous systems," SIAM Journal on Control and Optimization, vol. 38, no. 3, pp. 751-766, 2000.

[30] Y. Su, "Global continuous finite-time tracking of robot manipulators," International Journal of Robust and Nonlinear Control, vol. 19, no. 17, pp. 1871-1885, 2009.

[31] Y. Su and C. Zheng, "Global finite-time inverse tracking control of robot manipulators," Robotics and Computer-Integrated Manufacturing, vol. 27, no. 3, pp. 550-557, 2011. 


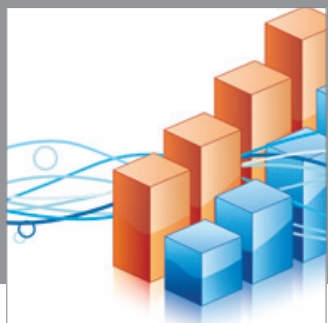

Advances in

Operations Research

mansans

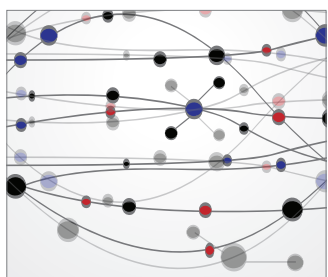

The Scientific World Journal
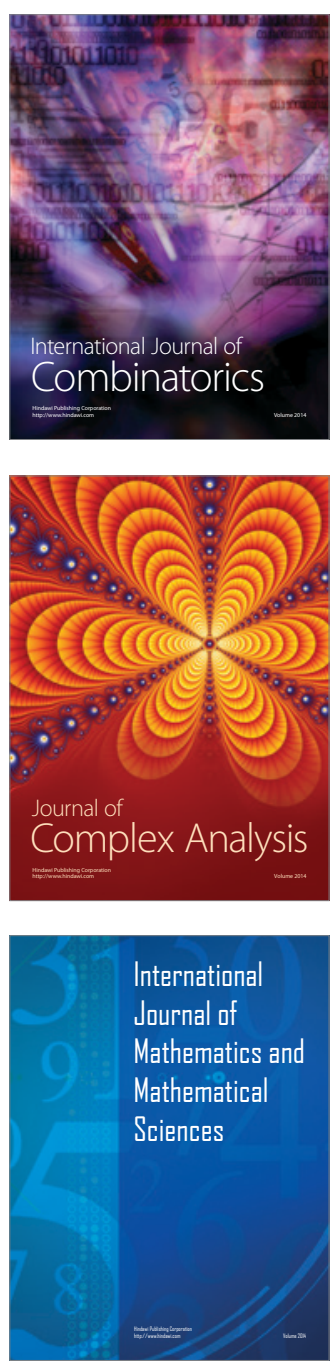
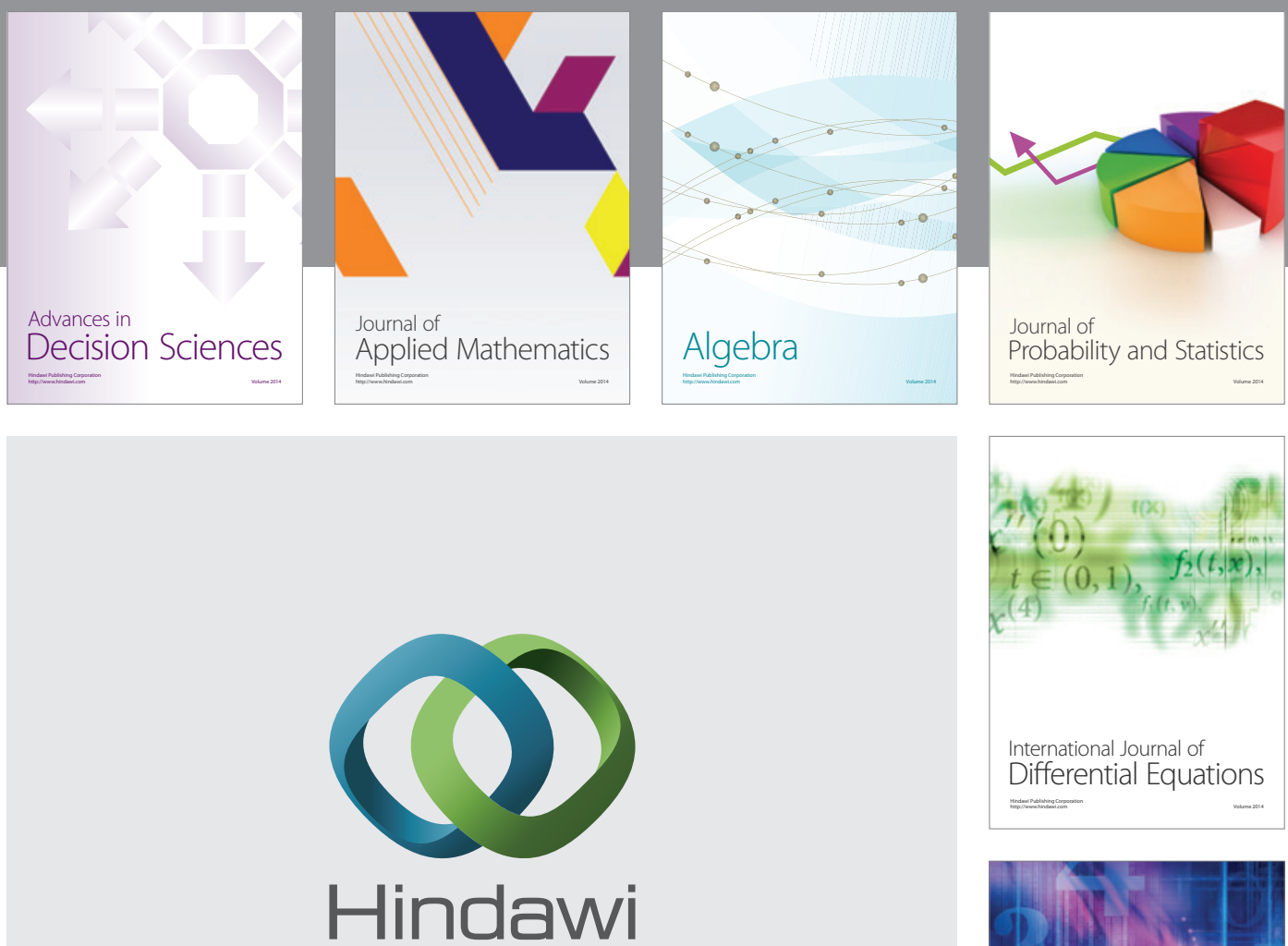

Submit your manuscripts at http://www.hindawi.com
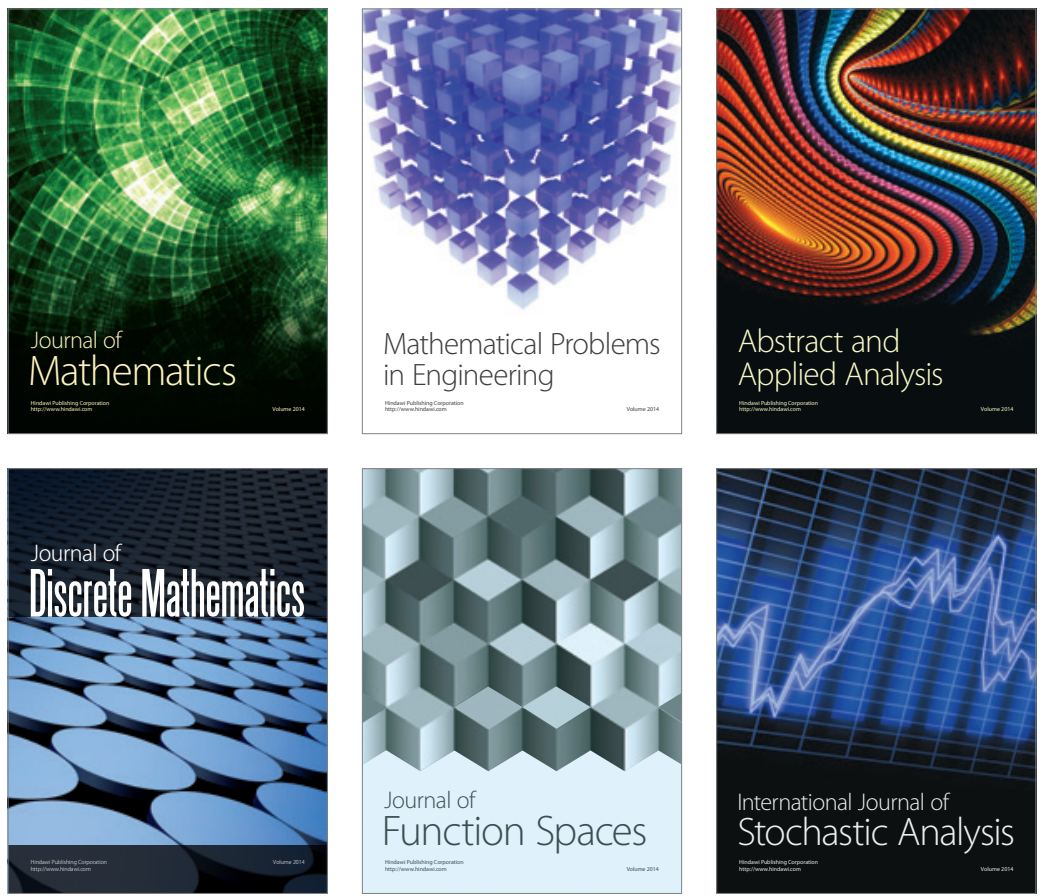

Journal of

Function Spaces

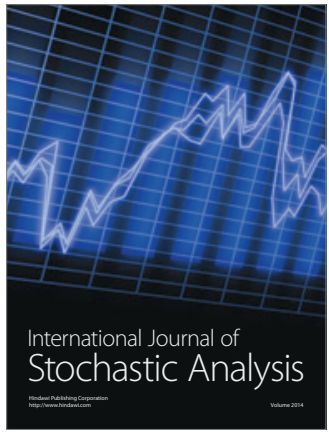

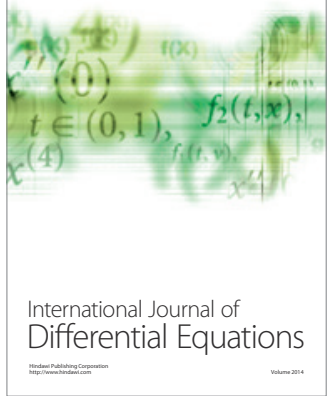
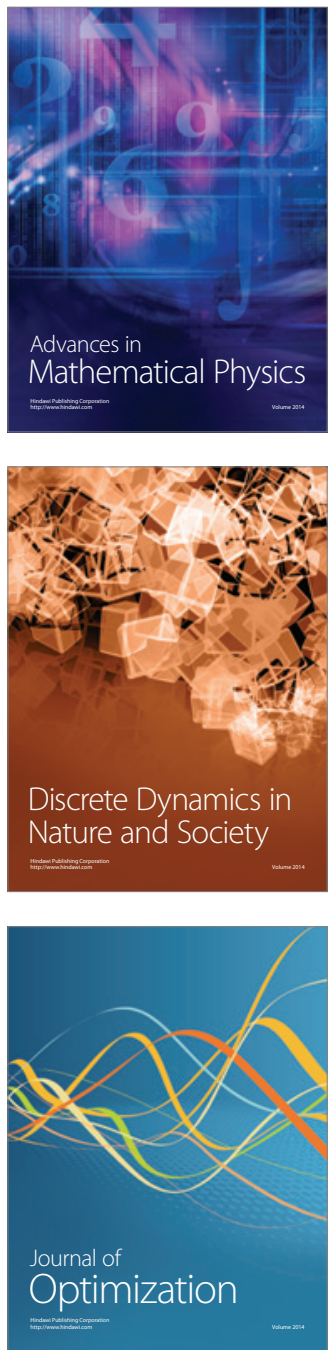